\title{
Simulation of a Two-Phase Composite Material with a System of Fibers Disoriented in Space
}

\author{
E.M. Romanovskaia, ${ }^{1, a)}$, E.A. Mityushov ${ }^{1, b)}$, S.A.Berestova, ${ }^{1, c)}$, \\ N.D. Romanovskaia ${ }^{1, d)}$ \\ ${ }^{1}$ Ural Federal University, Mira 19, Yekaterinburg, Russia, 620002 \\ a)Corresponding author: E.M.Romanovskaia@urfu.ru \\ b)mityushov-e@mail.ru \\ c)s.a.berestova@urfu.ru \\ d)NDRomanovskaya@yandex.ru
}

\begin{abstract}
The proposed method is based on the generally accepted operation of averaging properties at different orientations of the axes of elastic symmetry of the material in the areas, each of which is a unidirectional monotropic material. At the same time, statistical characteristics of fiber distribution in the composite material are introduced texture parameters that uniquely determine the elastic properties of the material. As a result, it is possible to obtain the average values of the elastic characteristics of the spatially reinforced composite material, linearly dependent on the elastic characteristics of the unidirectionally directed composite material and texture parameters for any fiber distribution in the material under the restriction that the material is orthotropic. Similar expressions are obtained for the mean compliance coefficients.

Keywords: composite material, effective characteristics, elasticity, elastic properties anisotropy, fibers, macroscopic modulus of elasticity, transversely isotropic material, spatially reinforced composite material, texture parameters, young's modulus, method of generalized self-consistent field
\end{abstract}

\section{INTRODUCTION}

Currently, more and more attention is attracted to composite materials with a spatial arrangement of reinforcement. The introduction of a spatial framework increases the bearing capacity of the material in thick-walled structures, especially in areas of concentrated loads, cuts, ribs with non-stationary force and temperature effects characteristic of modern technology. The presence of reinforcement with different stiffness and strength significantly expands the range of properties of composite materials with a spatial reinforcement scheme. The expansion of the use of spatially reinforced materials makes it necessary to obtain reliable numerical estimates of the physical and strength properties of these materials.

\section{STATEMENT OF THE PROBLEM AND THE METHOD OF SOLVING IT}

As a model, we take a fibrous material consisting of a matrix and reinforcement fibers. The fibers in the isotropic matrix are oriented chaotically and have isotropic or transversally isotropic properties. Three orthogonal planes of elastic symmetry pass through each point of the material, so the composite is orthotropic and is characterized by nine independent elastic constants. It is necessary to obtain expressions for macroscopic modulus of elasticity and malleability of such composite material. 


\section{ELASTICITY MODULES OF A SPATIALLY REINFORCED COMPOSITE}

Elastic modulus and compliance coefficients relate stresses to deformations. Stress $\sigma$ and deformation $\varepsilon$ are second-rank tensors whose components are in a rectangular Cartesian coordinate system $O x_{1} x_{2} x_{3}$ will be denoted by $\sigma_{i j}$ and $\varepsilon_{i j}$. Then, the elastic properties of the material are described by fourth-rank tensors of elastic modules $\mathrm{c}$ and stress coefficients s with components $c_{i j k l}$ и $s_{i j k l}$, respectively.

The generalized Hooke's law, which describes the elastic behavior of linearly elastic bodies, can be expressed both in terms of c and s: $\varepsilon=\mathrm{s} \sigma, \sigma=\mathrm{c} \varepsilon$, either component wise: $\varepsilon_{\mathrm{ij}}=\mathrm{s}_{\mathrm{ijkl}} \sigma_{\mathrm{kl}}, \sigma_{\mathrm{ij}}=\mathrm{c}_{\mathrm{ijkl}} \mathrm{s}_{\mathrm{kl}}$.

The tensors of elastic modules and stress coefficients are reciprocal.

To determine the properties of the material, we carry out the procedure of sequential averaging over phases and orientations. At the first stage, the effective elastic characteristics of the unidirectional fiber composite are determined by the method of generalized self-consistent field. The essence of the method [1] is to equalize the strain field in the fibers of a multiphase system, placed alternately in a homogeneous medium with the characteristics of a certain reference body, and the average field along the fibers of this phase in a heterogeneous system. General formulas for determining the characteristics of such a material are given in [2].

At the second stage, orientation averaging is performed, which corresponds to giving the system with parallel fibers all possible directions in space. At the same time, statistical characteristics of the distribution of fibers in the composite material are introduced - texture parameters $\Delta_{\mathrm{i}}$, which uniquely determine the elastic properties of the material. The average values of the $2 \mathrm{nd}$ and 4 th degrees of the cosines of the angles between the fiber axis and the laboratory coordinate system were selected as texture parameters.

As a result, using convolutions of the tensor of elastic characteristics for two indices, it was possible to obtain finite expressions for the average values of all elastic constants under the assumption that the composite material is orthotropic.

The dependences of the average flexibility modules of a composite material on the flexibility modules of a unidirectional composite and texture parameters will look like:

$$
\begin{gathered}
<s_{11}^{\prime}>=s_{11}-\left(2 s_{1}+s_{2}\right) \Delta_{1}+s_{3} \Delta_{4} \\
<s_{22}^{\prime}>=s_{11}-\left(2 s_{1}+s_{2}\right) \Delta_{2}+s_{3} \Delta_{5} \\
<s_{33}^{\prime}>=s_{11}-\left(2 s_{1}+s_{2}\right) \Delta_{3}+s_{3} \Delta_{6} \\
<s_{23}^{\prime}>=s_{13}+\left(s_{1}-s_{3}\right) \Delta_{1}+s_{3} / 2\left(1+\Delta_{4}-\Delta_{5}-\Delta_{6}\right) \\
<s_{13}^{\prime}>=s_{13}+\left(s_{1}-s_{3}\right) \Delta_{2}+s_{3} / 2\left(1+\Delta_{5}-\Delta_{6}-\Delta_{4}\right) \\
<s_{12}^{\prime}>=s_{13}+\left(s_{1}-s_{3}\right) \Delta_{3}+s_{3} / 2\left(1+\Delta_{6}-\Delta_{4}-\Delta_{5}\right) \\
<s_{44}^{\prime}>=s_{44}+\left(s_{2}-4 s_{3}\right) \Delta_{1}+s_{3} / 2\left(1+\Delta_{4}-\Delta_{5}-\Delta_{6}\right) \\
<s_{55}^{\prime}>=s_{44}+\left(s_{2}-4 s_{3}\right) \Delta_{2}+s_{3} / 2\left(1+\Delta_{5}-\Delta_{6}-\Delta_{4}\right) \\
<s_{66}^{\prime}>=s_{44}+\left(s_{2}-s_{3}\right) \Delta_{3}+s_{3} / 2\left(1+\Delta_{6}-\Delta_{4}-\Delta_{5}\right),
\end{gathered}
$$

where $\mathrm{s}_{1}=\mathrm{s}_{12}-\mathrm{s}_{13}, \mathrm{~s}_{2}=\mathrm{s}_{66}-\mathrm{s}_{44}, \mathrm{~s}_{3}=\mathrm{s}_{11}+\mathrm{s}_{33}-2 \mathrm{~s}_{13}-4 \mathrm{c}_{44}$, and also for unidirectional composite $\mathrm{s}_{11}-$ $\mathrm{s}_{12}=1 / 2 \mathrm{~s}_{66}$.

The resulting expression allows us to establish the elastic characteristics of a spatially reinforced composite by the elastic characteristics of a unidirectional reinforced material and well-defined texture parameters for any distribution of fibers in the material, with the restriction that the material is orthotropic.

\section{ANISOTROPY ANALYSIS OF YOUNG'S MODULUS}

As a rule, the anisotropy of the elastic properties of the material is associated with a change in the Young's modulus from the direction. The analysis of the anisotropy of the Young's modulus in composite materials with a spatially reinforced fiber system in any direction can be carried out by the values of texture parameters without involving cumbersome calculations and mechanical tests. To do this, consider the calculation of the module at an angle $\alpha$ to axis direction $0 \mathrm{x}_{3}$ in the plane $0 \mathrm{x}_{2} \mathrm{x}_{3}$.

The ratio can be obtained using the expression for the Young's modulus of an orthotropic material in a given direction $\alpha\left(\alpha_{1}, \alpha_{2}, \alpha_{3}\right)$ :

$$
\left.E^{-1}(\alpha)=s_{11} \alpha_{1}^{4}+s_{22} \alpha_{2}^{4}+s_{33} \alpha_{3}^{4}+\left(s_{\dot{4}}+2 s_{23}\right) \alpha_{2}^{4} \alpha_{3}^{4}+\left(s_{55}+2 s_{31}\right) \alpha_{3}^{4} \alpha_{1}^{4}+\left(s_{66}+2 s_{21}\right) \alpha_{1}^{4} \alpha_{2}^{4}\right)
$$

Assuming here $\alpha_{1}=0, \alpha_{2}=\sin \alpha, \alpha_{3}=\cos \alpha$, we get: 


$$
E^{-1}(\alpha)=s_{\dot{2}} \sin ^{4} \alpha+s_{33} \cos ^{4} \alpha+\left(s_{44}+2 s_{\dot{2} 3}\right) \sin ^{2} \alpha \cos ^{2} \alpha
$$

To illustrate the obtained relations, we present a calculation of the dependence of the Young's modulus on the direction of the measurement axis for a distribution with uniformly disoriented in the plane $0 \mathrm{x}_{1} \mathrm{x}_{2}$ fibers and fibers located near the axis direction $\mathrm{Ox}_{3}$ with an angle of $2 \theta^{\circ}$ (Fig. 1), for the material titanium - molybdenum.

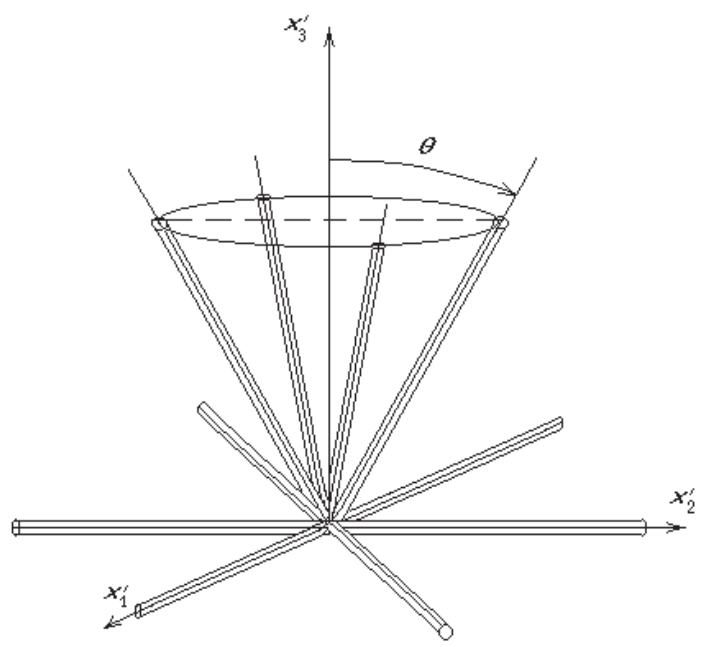

FIGURE 1. Distribution with uniformly disoriented in the plane of the fibers and fibers located on a conical surface.

The elastic phase characteristics are- Тi: $E^{m}=110 Г П а, v^{m}=0,36, \quad$ Mo: $E^{f}=320 \Gamma П a, v^{f}=0,305$. The concentration of fibers of the corresponding direction is- $v_{12}=0,2 ; v_{3}=0,1$ matrices $-v_{\mathrm{m}}=0,7$.

At the first stage, the characteristics of a unidirectionally reinforced composite were determined [2], while the fiber concentration was assumed to be equal to $v_{\mathrm{f}}=v_{12}+v_{3}=0,3$. At the second stage, the characteristics of the spatially reinforced material were calculated using formulas (1). Then, Young's modulus was studied using formula (2) depending on the direction of measurement.

For comparison, the anisotropy of the Young's modulus was calculated with a uniform distribution of fibers and composite with spherical inclusions for the titanium-molybdenum material by the method of generalized selfconsistent field, while the concentration of inclusions and matrix remained unchanged. The calculation results show that the anisotropy of Young's modulus is influenced by factors such as the nature of the texture, the scattering angle of the fibers, and the fiber concentration in the direction of the axes (Fig. 2).

By modeling the angle of deviation of the fibers from the vertical axis, it is possible to obtain a composite with the necessary properties. When comparing the anisotropy graphs of the Young's modulus for a composite reinforced with a fiber system according to the uniform distribution law and a composite with spherical inclusions with the same elastic characteristics, it is seen that the fibrous material has a greater rigidity of properties in all directions.

\section{CONCLUSION}

For materials with a spatially reinforced fiber system, a method has been developed for calculating the tensor of averaged ductility coefficients and elastic modules taking into account the system of texture parameters. For the materials under study, the anisotropy of Young's modulus was studied. 


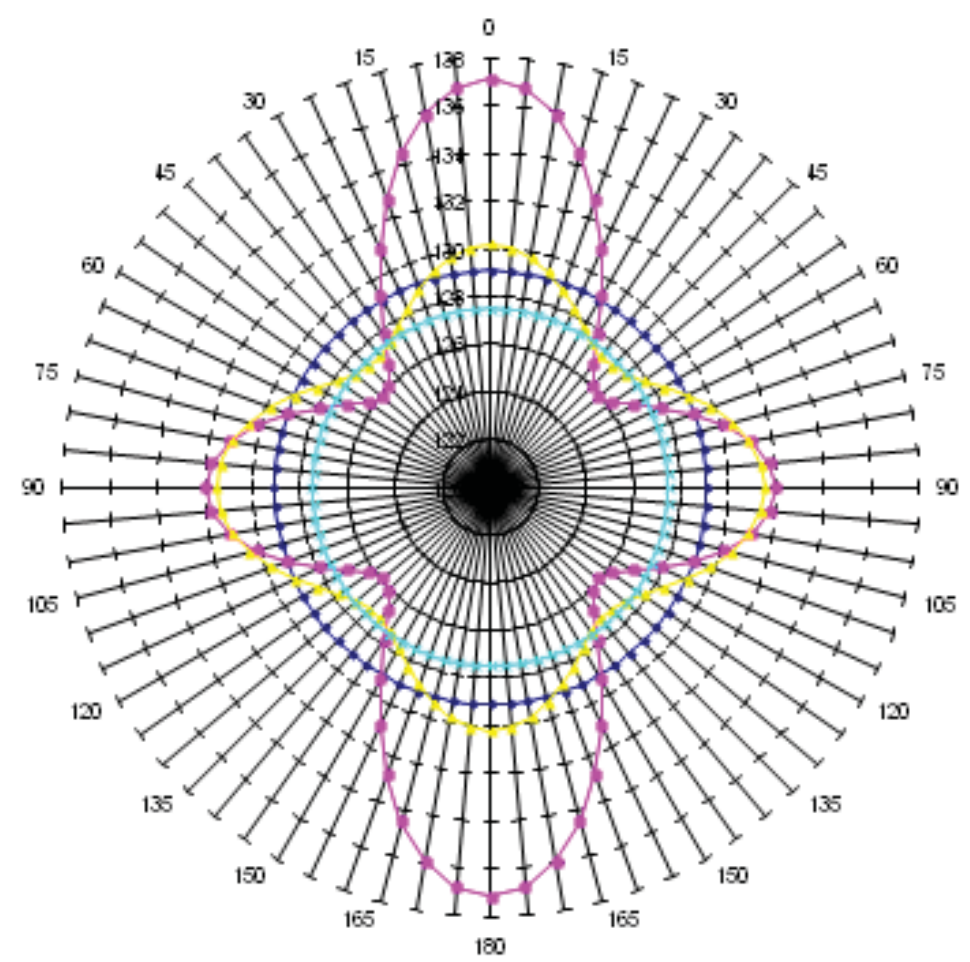

180

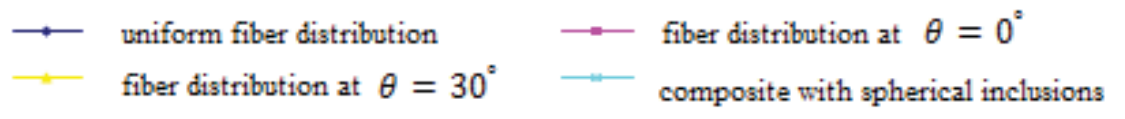

FIGURE 2. Anisotropy of Young's Ti-Mo modulus for various types of distributions.

\section{REFERENCES}

[1] E.A. Mityushov, P.V. Gel'd, R.A. Generalized conductivity and elasticity of macroscopical heterogeneous materials (Metallurgia, Moscow, 1992). (in Russian).

[2] E.M. Romanovskaia, E.A. Mityushov, S.A. Berestova, N.D. Romanovskaia, "Forecasting of the elastic properties of fibrous materials", AIP Conference Proceedings 2116, 430007 (2019); https://doi.org/10.1063/1.5114444. 